\title{
Using This Book
}

Italic bold signals a cross-reference. The glossary at the end lists some of the current English translations of Sanskrit terms that readers are likely to encounter. 